\title{
SPATIAL DOMAIN MITIGATION OF OUT OF BAND STRONG INTERFERERS IN HF WIDE BAND ACQUISITION USING ANALOG BEAMFORMING PRINCIPLES
}

\author{
S. Zazo*, I. Pérez-Álvarez ${ }^{\dagger}$, J. López-Pérez ${ }^{\dagger}$, B. Pérez-Díaz ${ }^{\dagger}$, E. Jiménez-Yguacel ${ }^{\dagger}$, \\ L. Melián-Gutiérrez ${ }^{\dagger}$, J.L. Sanz-González* \\ *Universidad Politécnica de Madrid (UPM), Dpto. SSR, E.T.S. Ingenieros de Telecomunicación, Ciudad Universitaria, 28040 \\ Madrid, Spain, e-mail: santiago@gaps.ssr.upm.es \\ $\dagger$ Instituto para el Desarrollo Tecnológico y la Innovación en Comunicaciones (IDeTIC), Universidad de las Palmas de G. C. \\ Campus de Tafira, 35017 Las Palmas G.C. Spain, e-mail: iperez@idetic.eu
}

Keywords: HF modem, Wideband Transmission, Interference Mitigation, Zero-Crossing Statistics, Analog Beamforming.

\begin{abstract}
We envision that dynamic multiband transmissions taking advantage of the receiver diversity (even for collocated antennas with different polarization or radiation pattern) will create a new paradigm for these links guaranteeing high quality and reliability. However, there are many challenges to face regarding the use of broadband reception where several out of band (with respect to multiband transmission) strong interferers, but still within the acquisition band, may limit dramatically the expected performance. In this paper we address this problem introducing a specific capability of the communication system that is able to mitigate these interferences using analog beamforming principles. Indeed, Higher Order Crossing (HOCs) joint statistics of the Single Input - Multiple Output (SIMO) system are shown to effectively determine the angle on arrival of the wavefront even operating over highly distorted signals.
\end{abstract}

\section{Introduction}

Our group has been working on HF transmissions for several years developing a proprietary system denoted as HFDVL (HF Data + Voice Links) that permits interactive digital voice transmission along with data communications reaching data rates up to $8640 \mathrm{bps}$ in the $3 \mathrm{kHz}$ bandwidth, as described in several publications (see for instance [8]). The performances of the modem have been improving over the last years, especially with the addition of multiple antennas at receiver, known as Single Input Multiple Output (SIMO) system [4, 6]. At this point we are dealing with a promising feature that uses much broader bandwidths than current systems. It is clear to us that in the near future much broader transmissions in the HF band will replace part of the current narrow band links.

We have noticed that some important steps have been taken in this direction. For instance, [2] describes some of the features of this tendency in the framework of the MIL-STD-188$141 \mathrm{C}$, which is likely to include specifications for HF radio passbands of (nominally) 6, 12, and $24 \mathrm{kHz}$ with data rates up to $64 \mathrm{Kbps}$. Also, there are already commercial transceivers including the 4-ISB mode as a transition phase to this concept.

Our personal view is that a real wide band signal is infeasible in this environment because the usage is typically very intensive and may suffer interferences from all over the world. Therefore we envision that as an alternative, dynamic multiband transmissions may provide a very satisfactory performance. In order to gain experience on this topic, we have built our own broadband transceiver [5], capable of transmitting simultaneously a set of up to 16 narrow band signals within $1 \mathrm{MHz}$ bandwidth where the number and positions of the channels depend on the current status of the propagation characteristics and the interference pattern. Very remarkably, we have observed that interferences out of our multiband but within the acquisition bandwidth degrade performance.

At first, this was somewhat unexpected until we realized that this effect comes from the reduction on the effective number of bits used to represent the desired signal because the Automatic Gain Control (AGC) intends to accommodate the aggregate received power in the ADC (Analog to Digital Converter) dynamic range. The consequent increase of the quantization noise dominates the thermal noise component and makes the system occasionally infeasible.

As this effect is introduced before the ADC itself we can not resort to any digital process to mitigate it but it has to be done in the analog domain. Therefore, a mitigation structure is required that operates on severely saturated signals as the interference may be of much higher power. In this paper we address a procedure based on Higher Order Crossings (HOC) statistics [3] that preserve the signal structure and allows the estimation of phase shifts between two highly correlated antennas and consequently determine the angle of arrival of the interference. This information is used by an analog beamformer to select the optimum weights to mitigate the interference.

The SIMO Mitigation Phase Block is the core of this proposal with the final objective of providing the fastest estimation of the interference channel to properly select the weights of the analog beamformer in order to let the standard Receiver operate under desirable conditions. In another paper presented to this conference [9] we were focused on the application of 
SISO (Single Input - Single Output) systems where the frequency estimator tunes an analog variable notch filter that mitigates the interference. In that paper, an alternative SISO Mitigation Phase Block was proposed.

The paper is structured as follows: section 2 presents some mathematical analysis to get some intuition about the effect we are dealing with. Section 3 will present the Mitigation procedure we propose and in section 4 we present some simulations with captured real data. Finally, section 5 sets some conclusions and forthcoming work.

\section{Mathematical Analysis}

This section describes a model as a baseline to address the challenge of broadband acquisition. The general approach is described in Figure 1 showing schematically the complex base band spectra of involved signals in the range $[0, B] \mathrm{Hz}$ where for simplicity one desired signal and just one interferer are sketched:



$\mathbf{B}$

Figure 1. Simplified schematic view in broadband acquisition

This analysis is included in full detail in an accompanying paper [7] but some results are considered in this paper for completeness. Main involved parameters are the following: $B$ : acquisition bandwidth. We suppose that the original bandpass signal is shifted to generate a complex base band version.

$W=B / M$ : desired signal bandwidth. We suppose an integer ratio $(M)$ between the bandwidth acquisition and $W$ to simplify the decimation process. Indeed it is realistic in HF where typically $\mathrm{W}=3 \mathrm{kHz}$ and $M$ will represent the number of standard channels within the captured bandwidth.

$x(t)$ is the desired bandlimited signal characterized by a certain power $\left(\sigma_{x}^{2}\right)$ and a certain Peak to Average Ratio (PAR) denoted as $P A R_{x}$ and defined as:

$$
P A R_{x}=\frac{\max \left(\|\left. x(t)\right|^{2}\right)}{\sigma_{x}^{2}}
$$

$n(t)$ is the thermal noise characterized as an additive white (within the acquisition band) Gaussian noise with flat spectrum density $N_{0}$. Power becomes $\sigma_{n}^{2}=B N_{0}$.

$i(t)$ is the interference bandlimited signal characterized by a certain power $\left(\sigma_{i}^{2}\right)$ and a certain $P A R_{i}$. $r(t)=x(t)+i(t)+n(t)$ is the aggregated signal.

The definition of the SNR is measured within the signal bandwidth $W$.

$$
S N R=\frac{\sigma_{x}^{2}}{W N_{0}}=\frac{B \sigma_{x}^{2}}{W \sigma_{n}^{2}}
$$

Once we have the base band signal, the part of the receiver where we are currently interested can be represented schematically in the Figure 2:

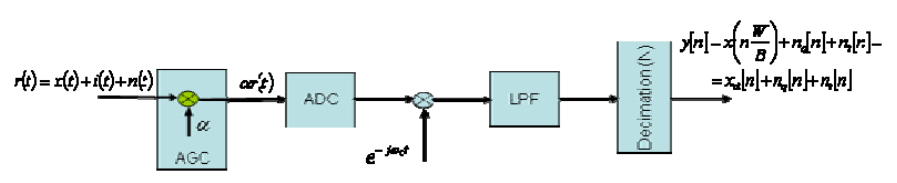

Figure 2. Standard broadband receiver (acquisition part)

In [7] it is shown that the SNR degradation suffered by a pure sinusoid desired signal (amplitude $A$ ) when an interference (also a sinusoid with amplitude $I$ ) is present can be approximated for different number of bits $(b)$ as follows:

$$
\begin{aligned}
\operatorname{SINR}(d B) & =S N R(d B)+10 \log _{10} \frac{3 A^{2} 2^{2 b} B / W}{3 A^{2} 2^{2 b} B / W+2 I^{2} 10^{\frac{S N R(d B)}{10}}}= \\
& =S N R(d B)-10 \log _{10}\left(1+\frac{2 I^{2} 10^{\frac{S N R(d B)}{10}}}{3 A^{2} 2^{2 b} B / W}\right)
\end{aligned}
$$

where SINR is the Signal to Noise + Interferences Ratio. Let us plot this function for different parameters in Figure 3:

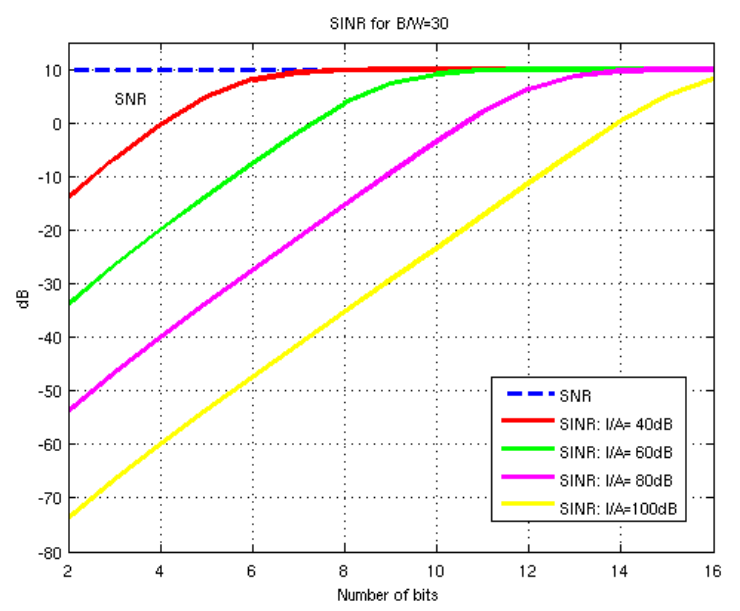

Figure 3. Performance degradation in the presence of interferences

Conclusions are quite clear: even if the receiver is equipped with a high quality ADC (12 - 14 bits) the degradation can be so dramatic that the communication will fail. In our opinion, future broadband HF communications of any nature will have to add some extra capabilities to deal with this problem.

\section{Mitigation procedure in the spatial domain}

In previous sections we have emphasized the effect of strong interferers within the acquisition band and the need to mitigate their effect. It is important to remark that we prefer to define this process as mitigation instead of cancellation because the final goal is to reduce the amount of interference and let the ADC work under proper conditions. If the signals are digitalized with the expected number of bits, all residual interferences will be eliminated by digital filtering. Therefore, 
the first issue to take into account is that this process has to be designed to operate before the signal digitalization.

In this paper we consider that we have a few antennas identical and quite close. In this case, optimum combination to increase the receiving diversity will provide minor improvement because the two signals are highly correlated. Another alternative in the presence of strong out of band (but still within the acquisition band) interferer that might make sense is to use these antennas to determine the angle of arrival of this interferer and mitigate it by proper weighting using analog beamforning principles.

Very importantly, we would like to stress that we are using real signal captured in Las Palmas de Gran Canaria (transmitted from Madrid) where three antennas are colocated on the roof of the building where our laboratory is located. Figure 4 shows the current deployment where two monopoles are separated by about 15 meters with the Yagi antenna in the middle.



Figure 4. Real deployment in ULPGC

We started measuring the correlation of these antennas and we confirmed what we have checked in other deployments [4, 6]. The Yagi antenna has a quite different radiation pattern and therefore is quite uncorrelated with respect to the other two, that are in fact quite correlated between them. So, in practice, one may use the Yagi and one monopole to optimally combine impinging signals. However, the study carried out in this paper will use the three of them: on one hand the Yagi and in the other a weighted combination of the two monopoles designed to mitigate the interference. Therefore, we achieve diversity gain and SNR improvement at the same time. Figure 5 shows the scheme we will study where this scenario adds an extra challenge: under standard conditions, the AGC of the receiver is matched to the desired signal so that to expand its dynamic range to the maximum number of bits of the ADC and therefore minimize the quantization effects. If the interference appears, typically with much larger power because the interference transmitter might be quite close to the desired receiver, the scenario changes instantaneously. As the AGC is designed to have some inertia and track channel response variations rate (few Hertz in HF), it is not capable to respond immediately to this new situation and thus the signal saturates (let us remark that even in the case we have an ideal AGC, this behaviour is not desirable because the number of bits dedicated to the desired signal will be fewer than needed and performance in any case degrade).



Figure 5. Receiver with the mitigation phase

Therefore, standard correlation procedures will be severely distorted because of the clipping effect at the ADC. In [9] we have shown that HOCs can be used to estimate the carrier frequency of a modulated signal and therefore, determine which channel is being used by the interferer. In this paper we apply a similar technique regarding phase shifts because this idea comes from the fact that HOCs [3] preserve most of the information embedded regarding frequencies and phases. So, the main point is that we are going to estimate the correlation (in amplitude and phase) using the highly saturated signals. In the end we will show that this statistical analysis is robust in these scenarios and will play an important role in this approach. Let us now describe in more detail the Mitigation Phase Block presented in Figure 5.

\subsection{Acquisition procedure}

The mitigation process will be accomplished in a separate digital domain in order to make simpler and efficient algorithms. Therefore, an extra ADC is needed. However, this device is not required to operate with similar number of bits as the ADC of the receiver. In this case, as the signal will be saturated, we just take care on the information related to the polarity to determine the zero-crossings. This extra ADC just need to provide a one bit signal to distinguish positive (1) and negative $(0)$ voltages. This sequence of $\{0 \mathrm{~s}, 1 \mathrm{~s}\}$ is all that is needed for the correlation estimation.

The acquisition block diagram is showed in Figure 6. Both receivers are frequency synchronized by a common external reference. Previous to the actual reception process the acquisition system is frequency and amplitude calibrated introducing a tone in both receivers. Finally it must be noted that both channels are digitalized in a synchronous way.

\subsection{Analog beamforming}

Very importantly, we can not think of generalized beamforming where the coefficients of the channels are estimated and optimally combined. In this scenario, the interference features are not accessible thus we must just resort on cross correlations between antennas in order to 
estimate the phase / amplitude shift between signals to align both components and increase / decrease the SNR. Regarding this problem, this cross correlation information will be used to mitigate as much as possible the interference effect. In this analysis, we will compare the mitigation capability when the full signals are acquired and also the related signals after saturation and conversion to a sequence of $\{0 \mathrm{~s}, 1 \mathrm{~s}\}$. We aim that this related signals preserve most of the information related to phase / amplitude shift and might be used for the mitigation process in comparison with the cross-correlation of the original signal as a benchmark.

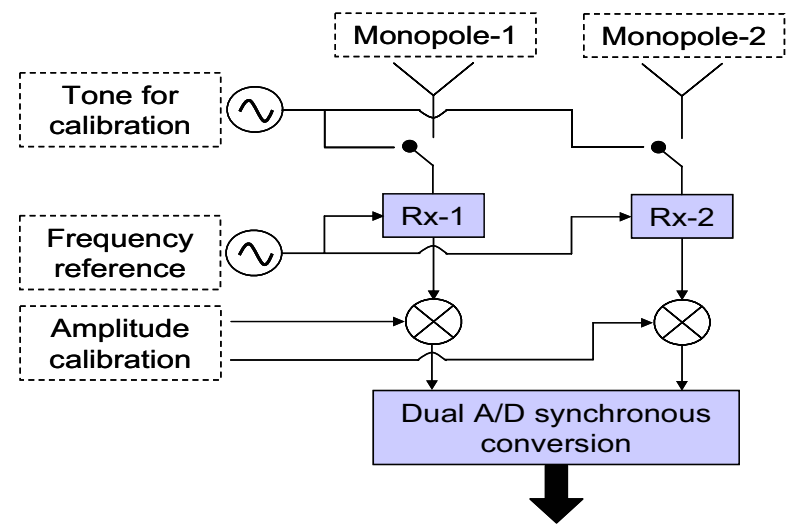

Figure 6. Acquisition block diagram

Once we have determined the suboptimum weights we will apply these coefficients to the analog beamformer. As in some cases, including amplitude information may be too expensive, we will also report results on the case where the beamformer uses only phase information.

Although we are quite far away of real implementations we have foreseen the state of the art regarding analog beamformers. Until very recently, it provided limited performance, specially because phase shifters tend to exhibit significant amplitude variations. However, some recent advances on SiCe-BICMOS technology, jointly with some innovative concepts introduced for phase and amplitude control circuits [2] have been made possible to develop a full $360^{\circ}$ control range of phase shifters together with amplitude control of more that $20 \mathrm{~dB}$. However, complexity, robustness and power dissipation are still an issue.

\section{Simulations with real data}

As already mentioned, we have transmitted from Madrid to Gran Canaria (a $1800 \mathrm{Km}$ link) an OFDM signal in $3 \mathrm{kHz}$ bandwidth using our HFDVL system. However, in this case we will not make use of any knowledge about its structure to obtain the correlation, instead we will consider the raw signal to emulate that a signal with unknown structured is received.

We would like to emphasize that the goal of this paper is not the full structure presented in Figure 5, but instead we will limit ourselves to the analog mitigation of one signal using the two monopoles.

The first objective is to evaluate the differences in terms of correlation when using the received non-clipped signals (labelled as "Full signal" in Figures 7 and 8) against the case when we are using the sequences of $\{0 \mathrm{~s}, 1 \mathrm{~s}\}$ of them (labelled as clipped signal in figures).

First of all, we noticed that the phase information shown in Figure 8 is nearly identical while respecting the amplitude both, original and clipped signals follow similar evolutions although there is some correlation loss due to the clipping effect (notice that each sample corresponds to the cross correlation coefficient calculated in a sliding window of 64 samples). It is worthy to mention that initially we expected a fixed coefficient but in practice we get a time dependent parameter due to the channel variations themselves.



Figure 7. Comparison of the amplitude of the correlation

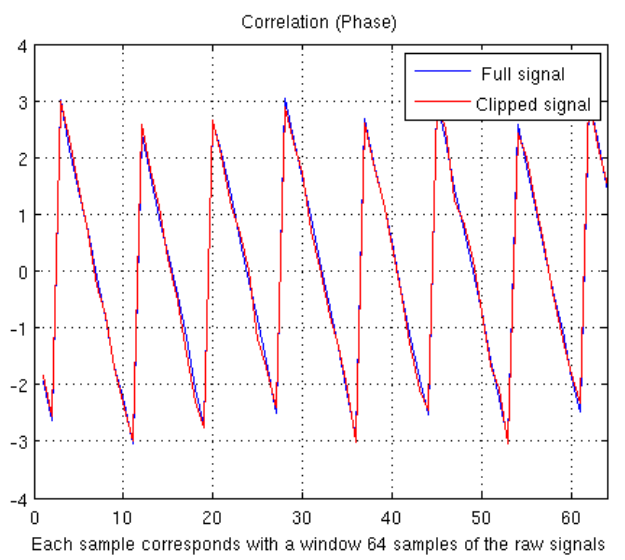

Figure 8. Comparison of the phase of the correlation

In a second stage we evaluate the percentage of power that we are able to cancel using complex analog beamformers (weights are defined by the amplitude and the phase) and also where pure delays (phase shifts) are applied. In both cases, we will compare the implementation where the full signal is available, Figure 9, against the case where just a clipped version of it, Figure 10.

It is important to remark that there is a minor improvement when using the full signal to estimate the cross correlation in comparison with that in the case where just clipped signals are used. This fact emphasizes the important role that HOCs may play in scenarios where strong saturation effects are caused. 
Also, it can be noticed that using just the phase information, that in turn will require simpler hardware to implement the beamformer, also a quite satisfactory result can be achieved.

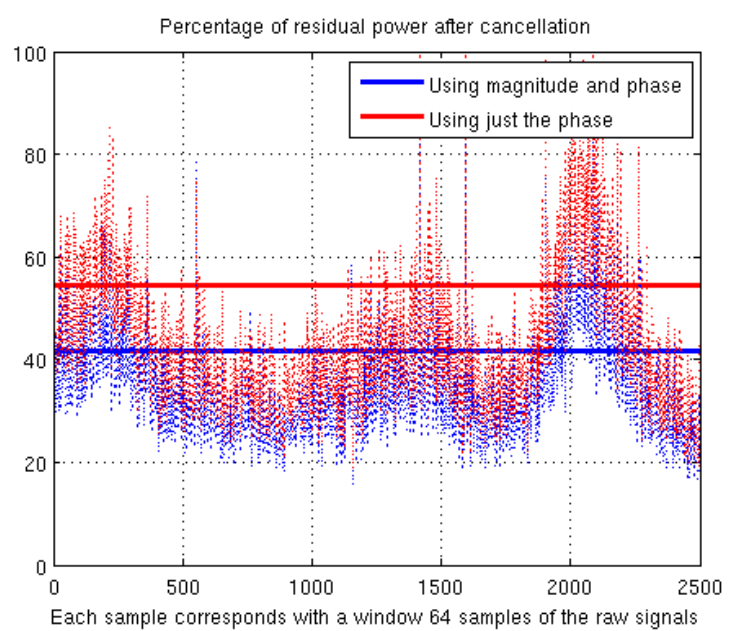

Figure 9. Residual energy using the full signals

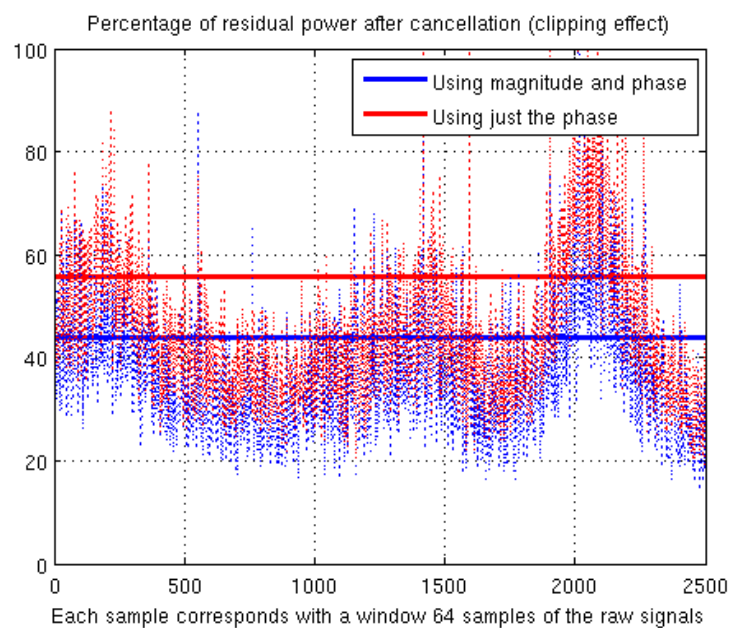

Figure 10. Residual energy using clipped signals

As shown in previous figures, the cancellation also depends on time, but most of the time we can guarantee more than 70 $\%$ cancellation for both the full and the clipped signal. Please, notice that we are talking about out of band interferers so we do not require complete elimination but just a mitigation that allows the ADC of the desired signals work properly in the joint receiver. Later on, in the digital domain, residual out of band signals might be filtered out in the frequency domain.

\section{Conclusions}

In this paper we have shown that broadband acquisitions might require an extra block specially designed to mitigate interferences within the bandwidth. We have highlighted that this procedure must operate in the analog domain and must be able to estimate the correlations among different antennas and implement an analog beamformer to mitigate its effect.
The scope of this paper has been to accomplish a preliminary study as a proof of concept using real data signals but simulating all the implementations. However, the outcome of the paper is quite promising and clearly shows that HOCs might be an important tool when operating with highly amplitude distorted signals. In the future we plan to build the hardware and real time software to show in real links these capabilities inside the HFDVL framework.

\section{Acknowledgements}

This work was supported by the Spanish Ministry of Science and Innovation under grants TEC2010-21217-C02 CR4HFDVL, and CONSOLIDER-INGENIO 2010 CSD2008-00010 COMONSENS.

\section{References}

[1] F. Ellinger. Radio frequency integrated circuits and technologies. Springer-Verlag. Berlin, 2007.

[2] E. E. Johnson. Performance Envelope of Broadband HF Data Waveforms, (WBHF Applications), Proceedings of MILCOM 2009, IEEE*, Boston, MA, Oct 2009.

[3] B. Kedem. Time Series Análisis by Higher Order Crossings. IEEE Press, New York 1994.

[4] Peco, C.; Zazo, S.; Pérez-Álvarez, I.; López-Pérez, J. On the diversity in multiantenna HF communications, IRST 2009: 11th International Conference on Ionospheric Radio Systems \& Techniques. Edinburgh, U.K.; April 2009.

[5] Pérez-Díaz, B.; Jiménez-Yguacel, E.; López-Pérez, J.; Pérez-Álvarez, I.; Zazo-Bello, S.; Medieta-Otero, E. Design and construction of a broadband $(1 \mathrm{MHz})$ digital $\mathrm{HF}$ transceiver for multicarrier and multichannel modulations, IRST 2009: 11th International Conference on Ionospheric Radio Systems \& Techniques. Edinburgh, U.K.; April 2009.

[6] Pérez-Álvarez, I., S. Zazo, M. Ghogho, J. López-Pérez, Experimental Results on Multicarrier MIMO HF Communications". 2011 URSI General Assembly and Scientific Symposium (GASS), Istanbul, Turkey, August 13-20, 2011.

[7] Pérez-Díaz, B., S. Zazo, I. Pérez-Álvarez, J. López-Pérez, L. Melián-Gutiérrez, E. Jiménez-Yguacel. Theory and Practice for Modelling the Broadband Acquisition in HF Transmissions. IRST 2012: 12th International Conference on Ionospheric Radio Systems \& Techniques. York, U.K.; May 2012.

[8] Santana, H.; Zazo, S.; Pérez, I.; Raos, I.; Mendieta, E.; López, J. Validation of a HF Spread Spectrum MultiCarrier Technology through real Link Measurements. European Transactions on Telecomunications.Vol.: 17, pp. 651- 657 Nov.-Dec 2006.

[9] Zazo,S., J.L. Sanz-González, B. Pérez-Díaz, I. PérezÁlvarez, J. López-Pérez, L. Melián-Gutiérrez. Analog mitigation of out of band strong interferers in wide band acquisition for multiband HF transmissions. IRST 2012: 12th International Conference on Ionospheric Radio Systems \& Techniques. York, U.K.; May 2012. 